\title{
A Method for Extending the Bandwidth of Metamaterial Absorber
}

\author{
Hong-Min Lee and Hyung-Sup Lee \\ Department of Electronic Engineering, Kyonggi University, Kyonggi-do, Suwon 443-760, Republic of Korea \\ Correspondence should be addressed to Hong-Min Lee, hmlee@kyonggi.ac.kr
}

Received 13 September 2012; Accepted 28 October 2012

Academic Editor: Alejandro Lucas Borja

Copyright ( $\odot 2012$ H.-M. Lee and H.-S. Lee. This is an open access article distributed under the Creative Commons Attribution License, which permits unrestricted use, distribution, and reproduction in any medium, provided the original work is properly cited.

\begin{abstract}
We present a method for extending the bandwidth of a metamaterial absorber (MMA) with multiresonance structure. The basic unit cell of the MMAs consists of a periodic arrangement of an electric-LC (ELC) resonator and a square loop structure. The absorption bandwidth of an MMA is effectively extended by combining five unit cell structures with different geometric dimensions into a coplanar unit cell. Experimental results show that the fabricated MMA is $0.8 \mathrm{~mm}\left(0.0026 \lambda_{0}\right)$ thick with a peak absorption rate of $93 \%$ at $10 \mathrm{GHz}$ and a full width at half maximum (FWHM) bandwidth of $970 \mathrm{MHz}$.
\end{abstract}

\section{Introduction}

Nowadays, the microwave absorbers are interested in military and civil application to reduce the electromagnetic interference among microwave components or electronic circuits mounted on the same platform. One of conventional absorbers is Salisbury screen, and its mechanism is explained well in [1]. This type of absorber involves the use of a resistive sheet and a metallic ground plane to cancel out reflections from the screen. Recently, absorber technology has seen several advancements with the use of artificially structured metamaterials (MTMs) for creating a high-performance absorber for the microwave, terahertz, and near infrared frequency regimes $[2-5]$. According to the effective medium theory, MTMs can be represented by the complex values of electric permittivity $\varepsilon_{\text {eff }}\left(=\varepsilon_{\text {eff }}^{\prime}+j \varepsilon_{\text {eff }}^{\prime \prime}\right)$ and magnetic permeability $\mu_{\text {eff }}\left(=\mu_{\text {eff }}^{\prime}+j \mu_{\text {eff }}^{\prime \prime}\right)$. This permittivity and permeability can be independently controlled by varying the dimensions of the electric and magnetic components. Additionally, by tuning the electric and magnetic resonances, an MTM can be impedance-matched to free space. As a result, 100\% absorbance is theoretically possible. Compared to the traditional microwave absorber, metamaterial structure can give some solutions to improve traditional absorber. First, the thickness of resonant metamaterial absorber (MMA) is much thinner than the traditional absorber which has quarter wavelength thickness [6]. Second, the fabrications of MMA are low cost and simple. However, most of the MMA structures are based on strong electromagnetic resonances to effectively absorb the incident electromagnetic wave; the absorbance bandwidth of MMA is quite narrow. This narrow bandwidth characteristic of MMA limits absorber applications, so the method for extending the absorbance bandwidth of MMA is needed. In recent years, MMAs with dualband [7] and multifrequency resonator $[8,9]$ have been reported, but these types of MMAs show multiple absorption peaks at the different frequency bands. In addition, the absorption bandwidth of these designed MMAs is narrow, typically no more than $10 \%$. This narrowband width feature limits the device applications of the resonant type of MMA.

In this paper, we propose an efficient method to extend the absorbance bandwidth of an MMA. By combining five unit cell structures with different geometric dimensions into a coplanar unit cell, we obtain the superposition of the five different absorption peaks. Various array configurations of five unit cells with different geometric dimensions are investigated in order to expend the bandwidth of MMA. The 3D field simulation tool, CST MWS (Micro Wave studio), is used for the design of the proposed MMA structure.

\section{The Design of an MTM Absorber Unit Cell}

Geometry of the proposed basic MMA unit cell is shown in Figure 1. The unit cell is designed on the front and back side 


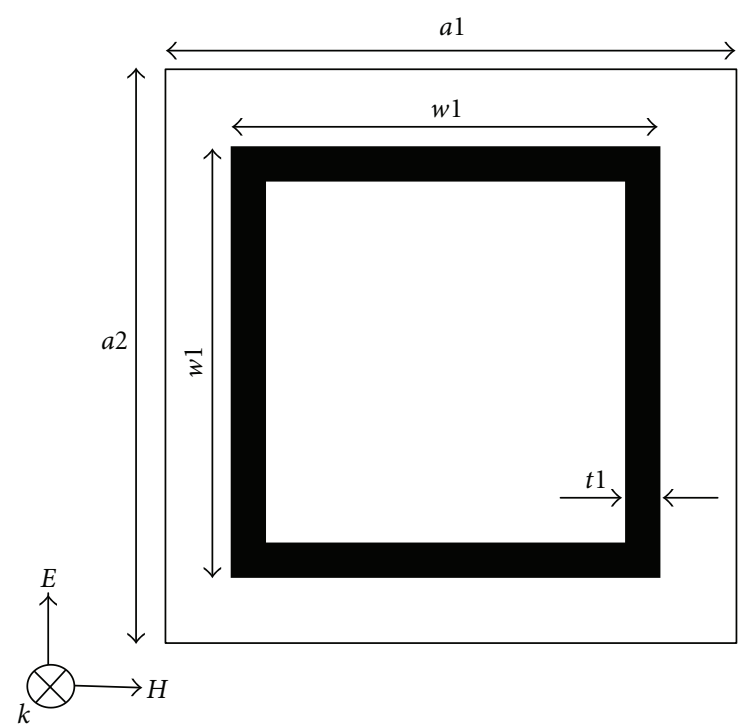

(a)

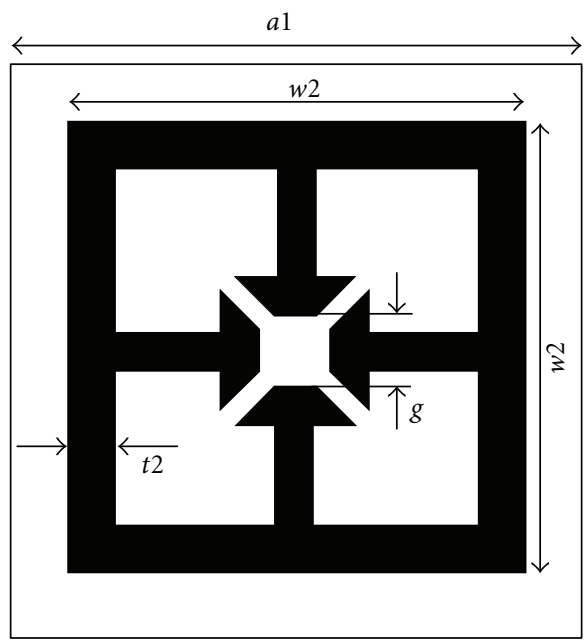

(b)

FIGURE 1: Geometry of the basic absorber unit cell $(a 1=5.8 \mathrm{~mm}$, $a 2=5.8 \mathrm{~mm}, w 1=4.1 \mathrm{~mm}, w 2=4.3 \mathrm{~mm}, g=0.9 \mathrm{~mm}, t 1=$ $0.3 \mathrm{~mm}, t 2=0.4 \mathrm{~mm}$ ); (a) front-view; (b) bottom-view.

of FR-4 substrate $\left(\varepsilon_{r}=4.6\right.$, loss tangent $\left.=0.025\right)$, and the thickness of FR- 4 substrate is $0.8 \mathrm{~mm}$. The unit cell consists of an electric-LC (ELC) resonator and a square loop element. Many kinds of ELC structure are reported in [10]. A polarization insensitive ELC structure, as shown in Figure 1(b), is selected in this design.

ELC resonator exhibits a resonant electric response when the electric field is polarized vertically or horizontally along the gaps of an ELC, and the circulating displacement currents which are generated between ELC and loop arms exhibit a resonant magnetic response. Overall size of the basic MMA unit cell is $5.8 \mathrm{~mm} \times 5.8 \mathrm{~mm} \times 0.8 \mathrm{~mm}$ including FR-4 substrate. The metallic pattern size of an ELC and a square loop structure are $4.3 \mathrm{~mm} \times 4.3 \mathrm{~mm}$ and $4.1 \mathrm{~mm} \times 4.1 \mathrm{~mm}$, respectively. The gap length of ELC structure is $0.9 \mathrm{~mm}$ and the width of two metallic patterns is $0.3 \mathrm{~mm}$.

We use frequency domain solver Microwave Studio by CST, and the program simulated a single unit cell with appropriate periodic boundary conditions (PBCs). A unit cell of the structure is placed inside a waveguide with $\mathrm{PBC}$ walls and a vertically polarized transverse electromagnetic (TEM) wave impinges upon this structure. The perfect electric conductor (PEC) boundary conditions are applied to the top and bottom walls of the waveguide, whereas perfect magnetic conductor (PMC) boundary conditions are applied to the side walls of the waveguide. The other two opposite sides of the waveguide are assigned as waveguide ports. The simulated frequency range is from $9.0 \mathrm{GHz}$ to $11 \mathrm{GHz}$. This structure generates antiparallel circulating current between the vertical arms of the ELC and the loop resulting in magnetic response, as shown in Figure 2(d). By tuning each of the resonances it is possible to match the impedance to space and minimize the reflectance. Figure 2(e) shows the simulated power loss densities in the ELC resonator structure at $10.12 \mathrm{GHz}$. The strongest electromagnetic energy absorption can take place where the electric field is confined in the vicinity of the dielectric substrate. This result indicates that the absorption does not homogeneously occur in the MTM unit cell.

Figure 3 shows the simulation results for the proposed MTM absorber, through the plotting of absorption rate $A(\omega)$, reflectance $R(\omega)$, and transmission $T(\omega)$. The scattering parameters of this MTM unit cell were simulated, and the absorption was calculated by the equation $A=1-$ $\left|S_{11}\right|^{2}-\left|S_{21}\right|^{2}=1-R(\omega)-T(\omega)$. It can be observed that the reflectance and transmission of the absorber sharply reduce to a minimum at $10.12 \mathrm{GHz}$, which also shows a peak absorption rate of $92 \%$. Based on absorption rate of $80 \%$, it has bandwidth of $220 \mathrm{MHz}$ and the full width at half maximum (FWHM) bandwidth of $425 \mathrm{MHz}$.

In Figure 2, we present the distributions of the simulated electric field for both TE (Figure 2(a)) and TM polarizations (Figure 2(b)), and magnetic field (Figure 2(c)), along with the surface current (Figure 2(d)) for the proposed MTM absorber unit cell in normal incidence at resonance frequency of $10.12 \mathrm{GHz}$. From the electric field distribution shown in Figures 2(a) and 2(b), one can observe that the four orthogonal gaps of the ELC element can confine the electric field owing to accumulated opposite charges both TE and TM polarizations. Hence, capacitance is produced in these gaps and the ELC element acts as an electric resonator. Meanwhile, the induced magnetic field for TE polarization is stronger in the regions close to the vertical arms of the ELC, as shown in Figure 2(c).

\section{Arrangement Configuration for Extending Bandwidth of MMA Cells}

The feasibility of a broad bandwidth MMA can be achieved by overlapping the absorption peaks of the five cells when their peaks closed to each other. To extend the bandwidth of MMA five MTM unit cells with different geometric 


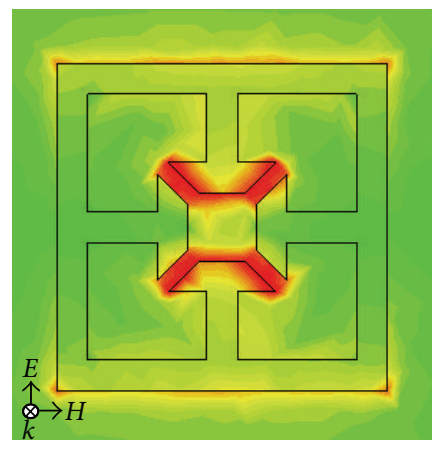

(a)

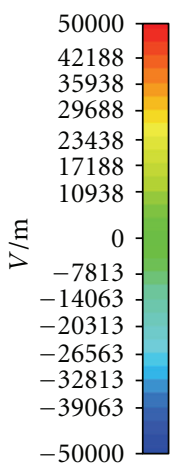

$-50000$

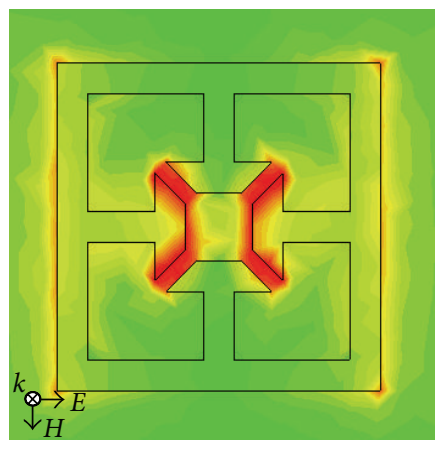

(b)
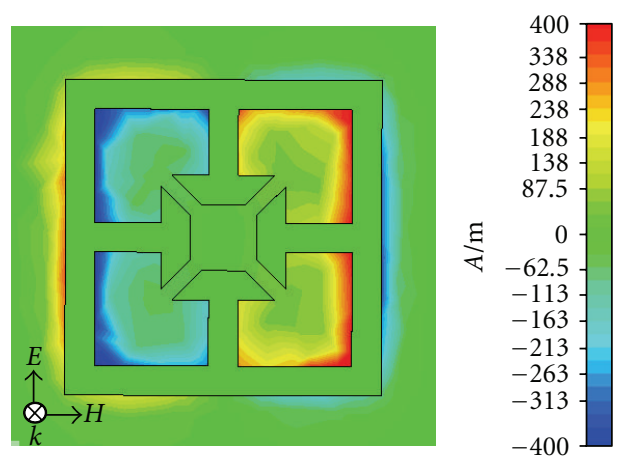

(c)
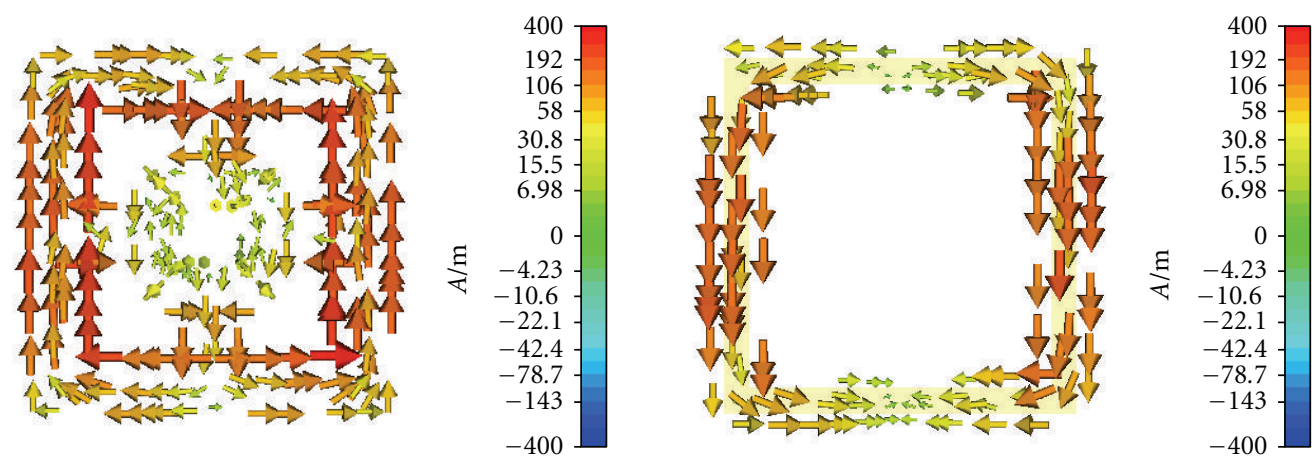

(d)
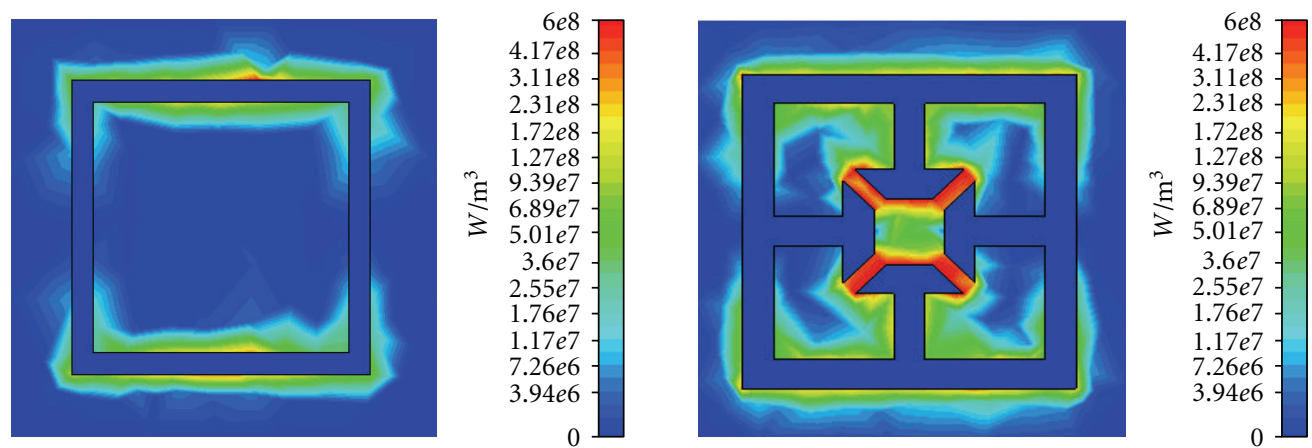

(e)

Figure 2: Simulated results of the proposed unit cell at $10.12 \mathrm{GHz}$; (a) electric field distribution for TE incidence; (b) electric field distribution for TM incidence; (c) magnetic field distribution for TE incidence; (d) surface current distribution for TE incidence; (e) the simulated average power loss densities of the absorber unit cell at two resonant frequencies. 


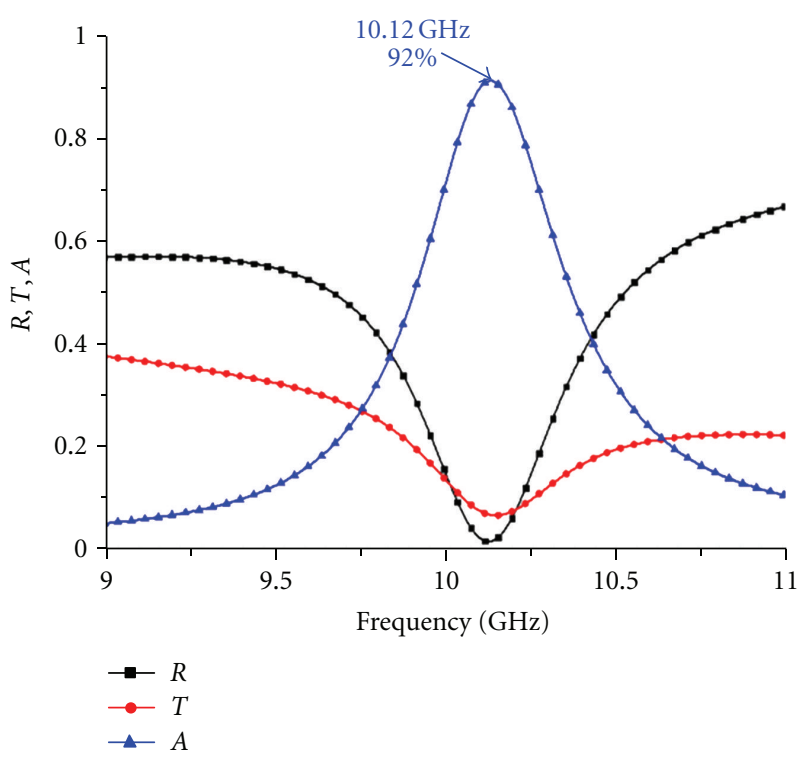

Figure 3: Calculated transmission $(T)$, reflection $(R)$, and absorption $(A)$ of the basic MMA unit cell from simulated $S$-parameters.

TABLE 1: Geometrical parameters of five different unit cells.

\begin{tabular}{lcccccccc}
\hline \multirow{2}{*}{ Cell type } & \multicolumn{8}{c}{ Geometrical parameters $(\mathrm{mm})$} \\
& $a 1$ & $a 2$ & $w 1$ & $w 2$ & $g$ & $t 1$ & $t 2$ & \multirow{2}{*}{$f_{0}(\mathrm{GHz})$} \\
\hline $\mathrm{K} 1$ & 5.8 & 5.8 & 4.0 & 4.1 & 1.5 & 0.7 & 0.4 & 10.50 \\
$\mathrm{~K} 2$ & 5.8 & 5.8 & 4.0 & 4.2 & 1.1 & 0.4 & 0.4 & 10.47 \\
$\mathrm{~K} 3$ & 5.8 & 5.8 & 4.1 & 4.3 & 0.9 & 0.3 & 0.4 & 10.12 \\
$\mathrm{~K} 4$ & 5.8 & 5.8 & 4.2 & 4.4 & 0.6 & 0.2 & 0.4 & 9.96 \\
$\mathrm{~K} 5$ & 5.8 & 5.8 & 4.2 & 4.4 & 0.7 & 0.2 & 0.4 & 9.69 \\
\hline
\end{tabular}

dimensions were designed. We select five different resonant unit cells with different scaling factors as shown in Table 1 and simulate various array configurations of five unit cells with a different arrangement, as shown in Table 2. We define the type of $\mathrm{K} 3$ as the basic unit cell sample, and some geometric dimensions of other four units are scaled up or down, as shown in Table 1. In addition, we also simulate the five single unit cells by changing the periodic array direction of the unit cells.

Figure 4 shows the calculated absorption characteristics comparison of five different single unit cells with different arrangement and array directions. To overlap the single resonant absorption peak of five single unit cells with different dimensions, the five single unit cells are periodically arrayed in order of K1-K2-K3-K4-K5 (Case 1), K2-K5-K3-K1-K4 (Case 2), and K5-K2-K1-K3-K4 (Case 3), respectively.

The simulated results show that the absorption bandwidth of the horizontal array configurations (cells are arrayed in $x$-axis) is narrow compared to vertical array configurations (cells are arrayed in $y$-axis) except in Case 2. Although peak absorption value of vertical arrayed unit cells in Case 1 is slightly lower compared to that of in Case 3 configurations, it shows the widest absorption bandwidth. The FWHM

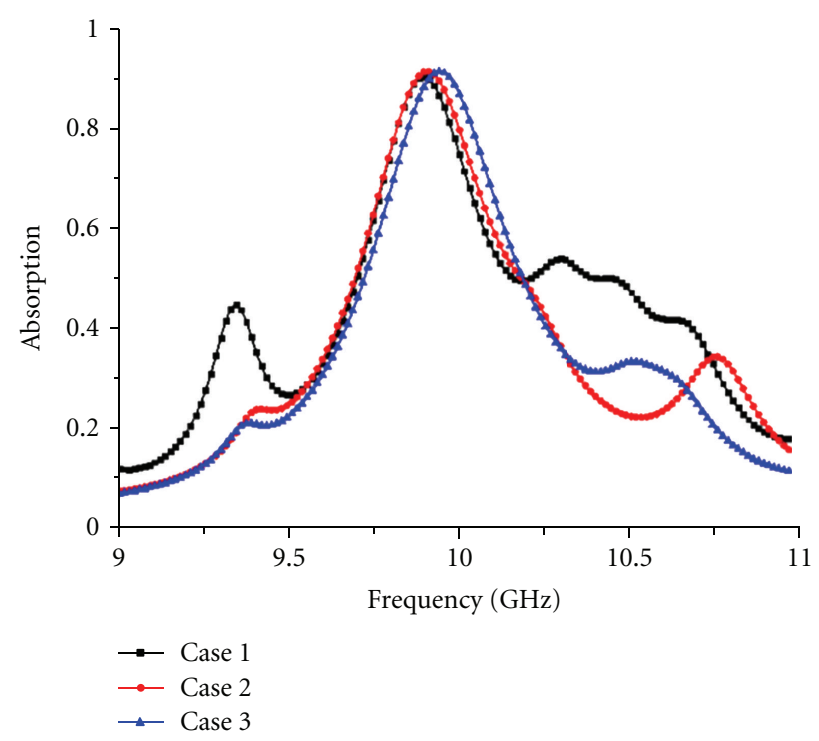

(a)

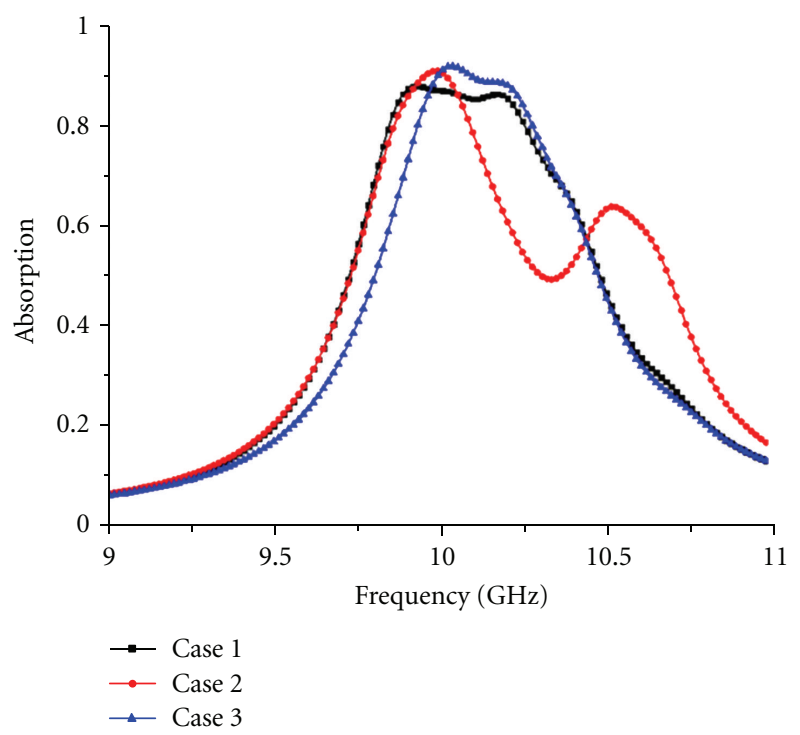

(b)

FIGURE 4: Comparison of the calculated absorption characteristics with five different single unit cells with different arrangement and array directions: (a) horizontal array; (b) vertical array.

TABle 2: Three different types of arrangement with five different unit cells.

\begin{tabular}{lc}
\hline Type & Arrangement \\
\hline Case 1 & K1-K2-K3-K4-K5 \\
Case 2 & K2-K5-K3-K1-K4 \\
Case 3 & K5-K2-K1-K3-K4 \\
\hline
\end{tabular}

bandwidth of vertical array configuration (Case 1) extended up to $770 \mathrm{MHz}$.

As the differences of scaling factors between cells are decreased, the five single resonant absorption peaks will overlap together. This is mainly due to the combination of 
five different geometric dimensions of unit cell results to shift of the resonance frequency to lower or higher values, depending on the capacitive or inductive nature of the coupling. When the five single unit cells are periodically arrayed in order of K1-K2-K3-K4-K5 (Case 1), it shows the smallest differences of scaling factors between cells. In this mixture of five unit cells with different geometric dimensions, the five single resonant absorption peaks will overlap together, and the bandwidth of an MMA is extended. On the other hand, increasing the differences of scaling factors between cells results to splitting of the five single resonant absorption peaks will single resonance into five distinct resonances. As a result, the five single unit cells are periodically arrayed in different order (Case 2 and Case 3), it shows the lowered absorption bandwidth compare to Case 1. Further broadening of the absorption bandwidth is also possible by increasing the number of different geometric dimensions of unit cell with proper combination.

Figure 5 shows the perspective view of the proposed MMA structure and the comparison of the calculated absorption for periodic array of single unit cell (K3 type) and five different single unit cells arrayed in vertical direction with different arrangement (Case 1). It is observed from Figure 5 that the absorption bandwidth of five different single unit cells array configuration increases compared to the single unit cell array configuration. The FWHM absorption bandwidth is extended from $470 \mathrm{MHz}$ to $770 \mathrm{MHz}$ by using five unit cell structures with different geometric dimensions. This extended absorption bandwidth of the MMA is mainly due to the electromagnetic field coupling between the neighbour unit cells [11]. Figure 6 shows the simulated electric field distributions of the five different absorber unit cells arrayed in vertical direction (Case 1).

At different resonant frequencies, two or three neighbouring unit cells exhibit strong electric field in the area between ELCs, indicating a capacitive coupling. As a result, the proposed MMA structure no longer shows singleresonant characteristics. By combining five unit cell structures with different geometric dimensions, some of resonant peaks closely positioned absorber unit cells are overlapped. Thus, the bandwidth of an MMA can be expended due to the multiresonance effect.

The nature of this absorption can be understood from Figure 7, which shows the simulated power loss densities of the absorber unit cell at three different resonant frequencies; 9.69, 10.12, and $10.5 \mathrm{GHz}$. At different resonant frequencies, the power loss is concentrated strongly in the vicinity of the open gaps and two or three neighbouring ELCs unit cells. In the proposed MMA structure, the strongest electromagnetic energy absorption, as shown in Figure 7, takes place where the electric field is confined in the vicinity of the dielectric substrate, as shown in Figure 6.

\section{The Fabricated Absorber Measurement Results}

We fabricated a prototype absorber for experimentation. Photographs of the fabricated two-layer metallization MTM

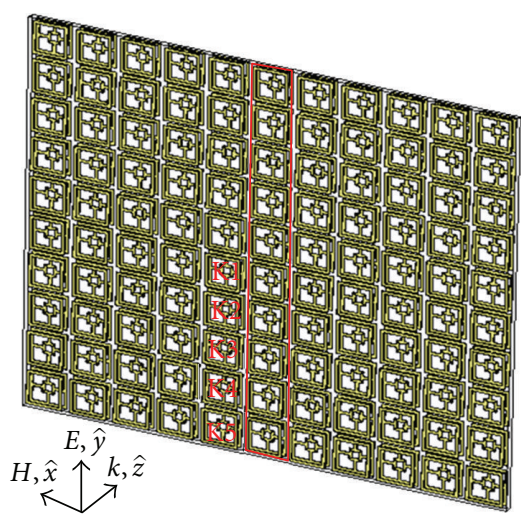

(a)

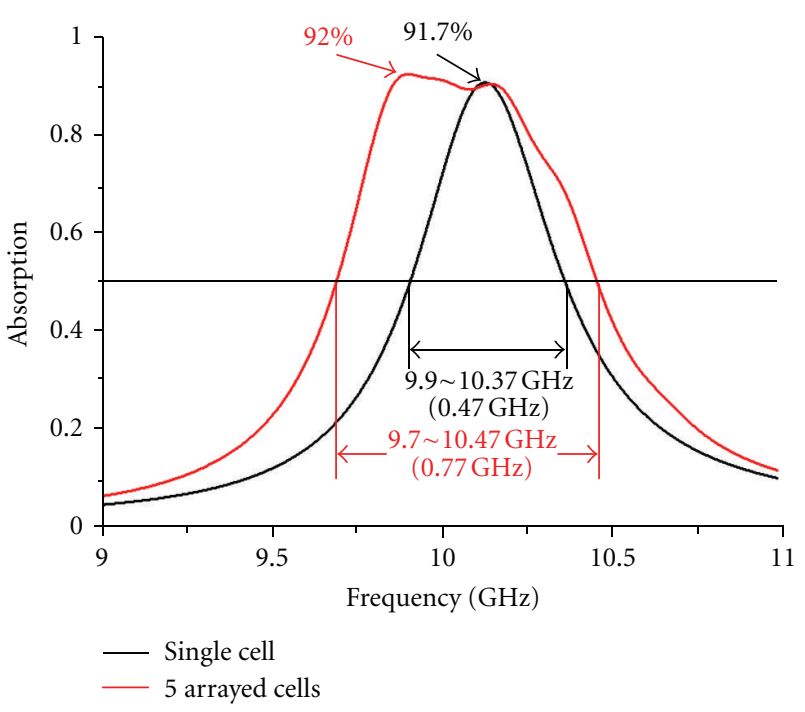

(b)

Figure 5: (a) Perspective view of the proposed MMA structure; (b) comparison of the calculated absorption for periodic array of single unit cell (K3 type) and five different unit cells arrayed in vertical direction.

absorber sample are shown in Figure 8. The sample was etched on an FR-4 substrate (relative dielectric constant $\varepsilon_{r}=$ 4.6, loss tangent $\delta=0.025$, and thickness $t=0.8 \mathrm{~mm}$ ) using standard photolithography techniques. In order to verify the effectiveness of the MTM absorber cells, a planar array of absorber unit cells $(40 \times 45)$ etched on both sides of an FR-4 substrate was mounted on an acryl substrate frame for measurement. The interelement spacing between the two absorber cells was set to $0.2 \mathrm{~mm}$, and the total size of the planar absorber was $243.6 \mathrm{~mm} \times 249 \mathrm{~mm}$. We experimentally verified the behaviour of the absorber by measuring the $S$-parameters of a planar array of unit cells. Figure 9 shows the schematic of the test setup for reflection and transmission measurement of the fabricated absorber sample.

We used a vector network analyzer and two X-band microwave rectangular horn antennas. Measurements were taken over a frequency range from 9 to $11.0 \mathrm{GHz}$. Before starting the characterization measurements, we performed 


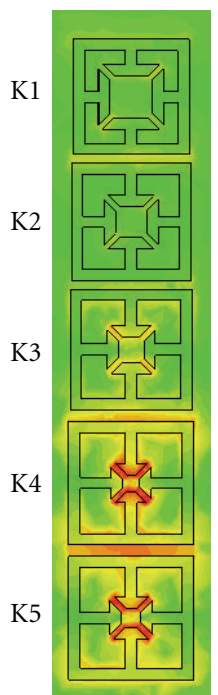

$9.6 \mathrm{GHz}$

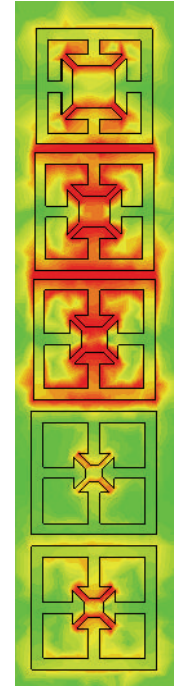

$10.12 \mathrm{GHz}$

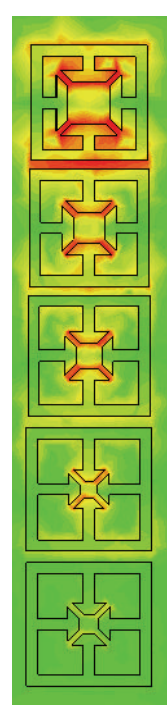

$10.5 \mathrm{GHz}$

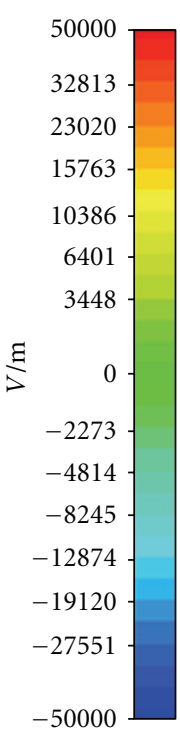

Figure 6: Simulated electric field distributions of the absorber unit cell at different resonant frequencies.

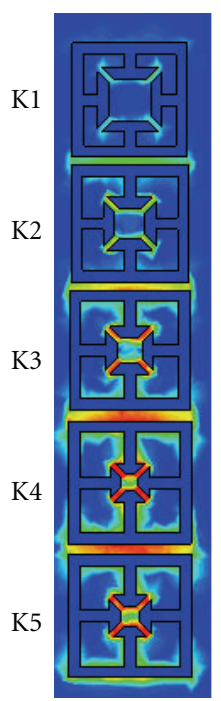

$9.69 \mathrm{GHz}$

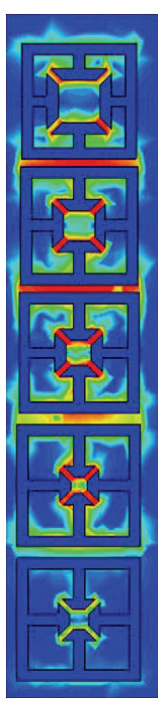

$10.12 \mathrm{GHz}$

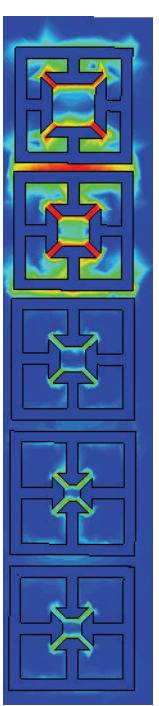

$10.5 \mathrm{GHz}$

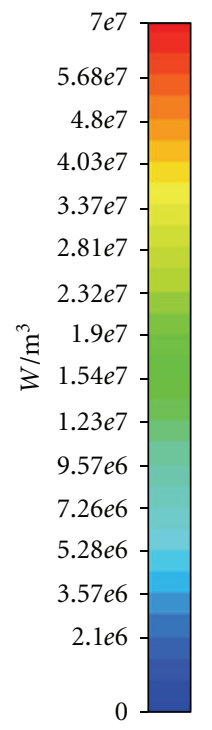

FIGURE 7: Simulated average power loss densities of the absorber unit cell at three different resonant frequencies.

a through-reflect-line (TRL) calibration. The reference plane is located at the surface of the sample. The reflection measurement has been calibrated by replacing the sample with an aluminum plate of same size at the reference plane. The transmission measurement has been calibrated with no sample.

As shown in Figure 9(a), a pair of horn antennas were used to transmit the EM wave on the sample absorber sheet and receive transmitted signals to obtain the $S_{21}$ parameters for normal incidence. Microwave absorbing material is placed between two horns and surrounding the sample sheet

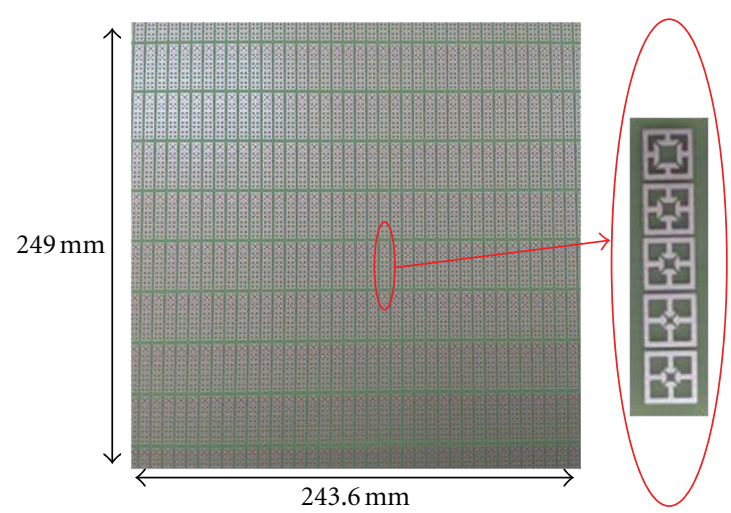

(a)

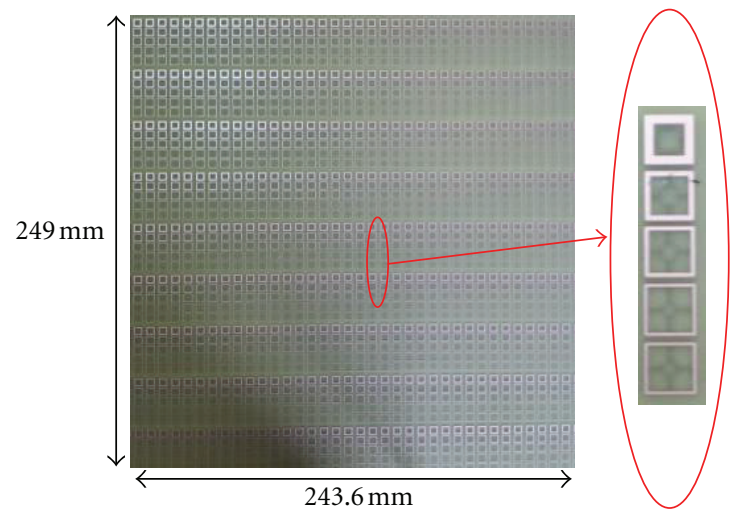

(b)

Figure 8: Photographs of the fabricated prototype absorber.

to eliminate unwanted edge scattering. To obtain the $S_{11}$ parameters, two horns were focused on the sample sheet on the same side, as shown in Figure 9(b). The height of the horn antennas is maintained at $1.2 \mathrm{~m}$ and the distance between horns and sample sheet is $0.6 \mathrm{~m}$ to eliminate nearfield coupling effects. Figure 10 shows a comparison of the simulated and measured absorption of the proposed MMA using measured magnitudes of the $S_{11}$ and $S_{21}$ parameters. A slight increment in the measured bandwidth in comparison with that of the simulated result is attributed to the fabrication tolerances and experiment setup for the proposed MMA. In addition, the experimental absorption frequency band is shifted approximately $60 \mathrm{MHz}$ higher when compared to the simulated results. The measured FWHM bandwidth is extended from $470 \mathrm{MHz}$ to $940 \mathrm{MHz}$ compared to the single unit cell array configuration. The proposed absorber shows a peak absorption rate of $93 \%$ at $10 \mathrm{GHz}$.

\section{Conclusion}

This paper presents an efficient approach for achieving the extended absorbance bandwidth of an MMA. The proposed MMA unit cells, which consist of an ELC resonator and a square loop structure, are etched on both sides of the FR-4 substrate. The simulated results of the basic unit cell show a peak absorption rate of $92 \%$ at $10.12 \mathrm{GHz}$, and FWHM 


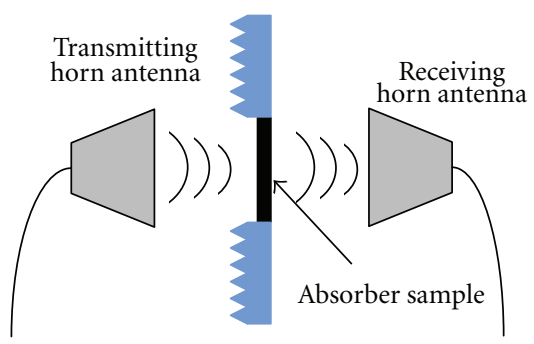

(a)

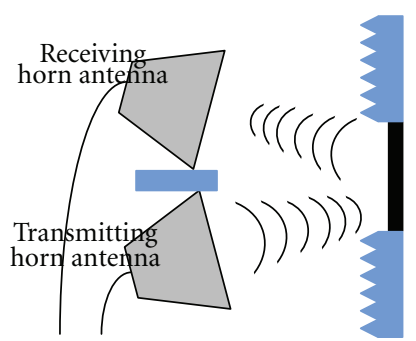

(b)

Figure 9: (a) Schematics of reflection and transmission measurement test setup for normal incidence and (b) oblique incidence.

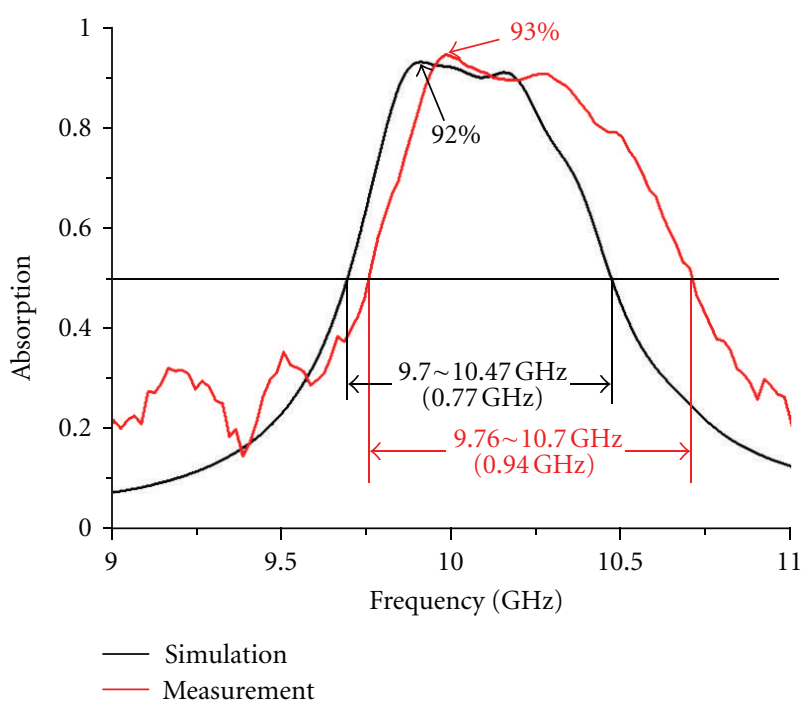

Figure 10: Comparison of simulated and experimented absorption of five unit cells with different dimensions arrayed in vertical direction (Case 1).

bandwidth of $470 \mathrm{MHz}$. By combining five unit cell structures with different geometric dimensions, their resonant peaks are overlapped. In addition, by placing the arrangement direction of the cells in vertically, the FWHM of the proposed MMA is extended up to $100 \%$ experimentally. This proposed configuration is an efficient method for extending the bandwidth of a MMA. The proposed layout can be easily extended to work for more compact, thinner backplaneless planar absorber designs for millimetre and terahertz frequency applications and can be applied for EM wave absorbing materials and microwave devices.

\section{Acknowledgment}

This research was supported by Basic Science Research Program through National Research Foundation of Korea (NRF) funded by the Ministry of Education, Science and Technology (no. 2010-0011646).

\section{References}

[1] R. L. Fante and M. T. McCormack, "Reflection properties of the Salisbury screen," IEEE Transactions on Antennas and Propagation, vol. 36, no. 10, pp. 1443-1454, 1988.

[2] N. I. Landy, S. Sajuyigbe, J. J. Mock, D. R. Smith, and W. J. Padilla, "Perfect metamaterial absorber," Physical Review Letters, vol. 100, no. 20, Article ID 207402, 4 pages, 2008.

[3] H. Tao, N. I. Landy, C. M. Bingham, X. Zhang, R. D. Averitt, and W. J. Padilla, "A metamaterial absorber for the terahertz regime: design, fabrication and characterization," Optics Express, vol. 16, no. 10, pp. 7181-7188, 2008.

[4] Y. Cheng and H. Yang, "Design, simulation, and measurement of metamaterial absorber," Journal of Applied Physics, vol. 108, no. 3, Article ID 034906, 4 pages, 2010.

[5] K. Boratay Alici, A. Burak Turhan, C. M. Soukoulis, and E. Ozbay, "Optically thin composite resonant absorber at the near-infrared band: a polarization independent and spectrally broadband configuration," Optics Express, vol. 19, no. 15, pp. 14260-14267, 2011.

[6] J. Lee, Y. J. Yoon, and S. Lim, "Ultra-thin polarization independent absorber using hexagonal interdigital metamaterials," ETRI Journal, vol. 34, no. 1, pp. 126-129, 2012.

[7] H. Tao, C. M. Bingham, D. Pilon et al., "A dual band terahertz metamaterial absorber," Journal of Physics D, vol. 43, no. 22, Article ID 225102, 2010.

[8] X. Shen, T. J. Cui, J. Zhao, H. F. Ma, W. X. Jiang, and H. Li, "Polarization-independent wide-angle triple-band metamaterial absorber," Optics Express, vol. 19, no. 10, pp. 9401-9407, 2011.

[9] H. Li, L. H. Yuan, B. Zhou, X. P. Shen, Q. Cheng, and T. J. Cui, "Ultrathin multiband gigahertz metamaterial absorbers," Journal of Applied Physics, vol. 110, no. 1, Article ID 014909, 8 pages, 2011.

[10] W. J. Padilla, M. T. Aronsson, C. Highstrete, M. Lee, A. J. Taylor, and R. D. Averitt, "Electrically resonant terahertz metamaterials: theoretical and experimental investigations," Physical Review B, vol. 75, no. 4, Article ID 041102, 4 pages, 2007.

[11] Y. Qiu Xu, P. Heng Zhou, H. Bin Zhang, L. Chen, and L. Jiang Deng, "A wide-angle planar metamaterial absorber based on split ring resonator coupling," Journal of Applied Physics, vol. 110, no. 4, Article ID 044102, 5 pages, 2011. 

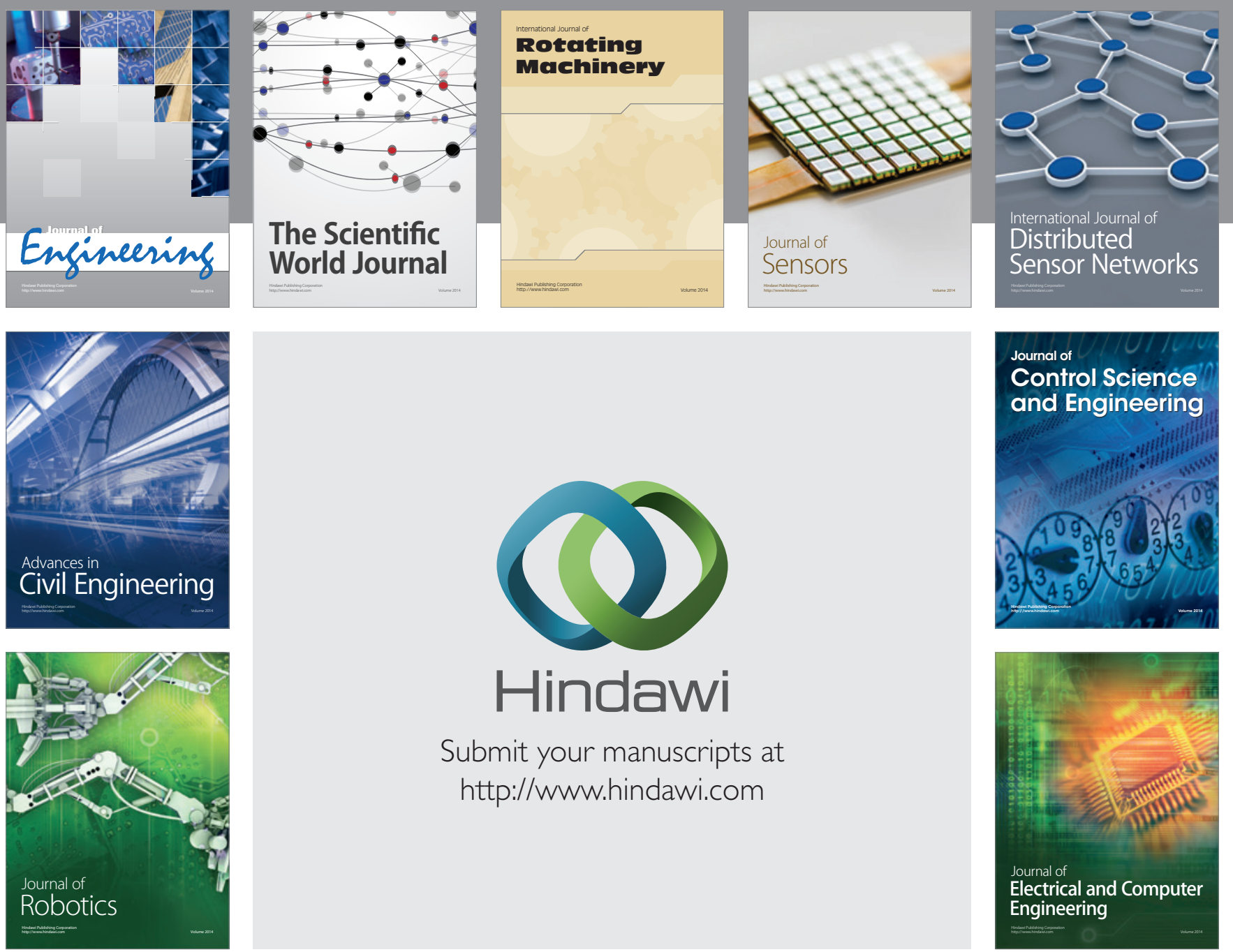

Submit your manuscripts at

http://www.hindawi.com
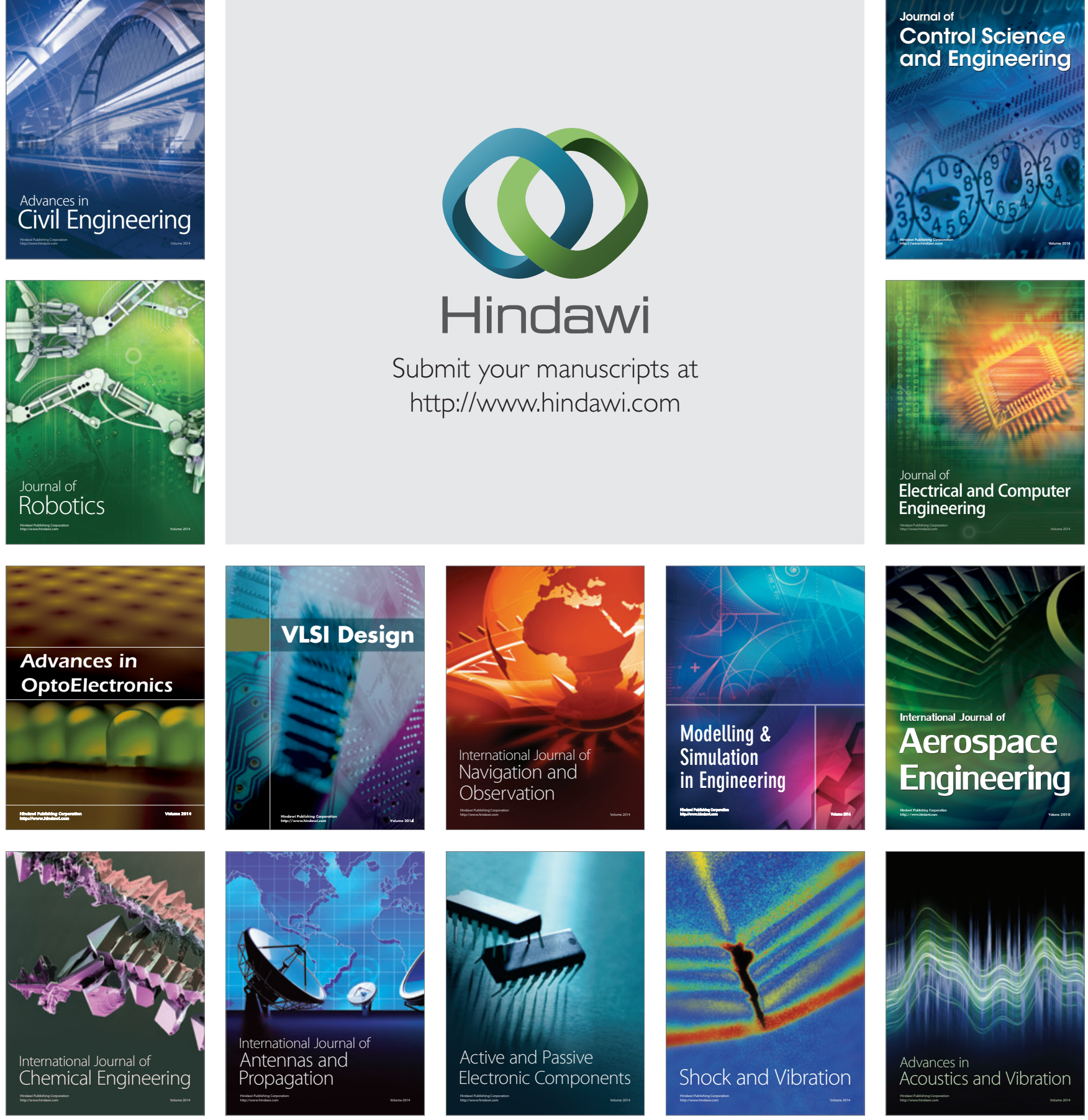